\title{
Chiral Anomalies in Even and Odd Dimensions
}

\author{
Jouko Mickelsson
}

Department of Mathematics, University of Jyväskylä, Seminaarinkatu 15, Jyväskylä 10, Finland, and Research Institute for Theoretical Physics, Siltavuorenpenger 20 C, Helsinki 17, Finland

\begin{abstract}
Odd dimensional Yang-Mills theories with an extra 'topological mass" term, defined by the Chern-Simons secondary characteristic, are discussed. It is shown in detail how the topological mass affects the equal time charge commutation relations and how the modified commutation relations are related to non-abelian chiral anomalies in even dimensions. We also study the SU(3) chiral model (Wess-Zumino model) in four dimensions and we show how a gauge invariant interaction with an external $\mathrm{SU}(3)$ vector potential can be defined with the help of the Chern-Simons characteristic in five dimensions.
\end{abstract}

\section{Introduction}

In a Yang-Mills theory in $2+1$ space-time dimensions one can add a "topological mass" term $\alpha \mathscr{L}_{\mathrm{cs}}^{(3)}$ to the Yang-Mills Lagrangian $\mathscr{L}_{\mathrm{ym}}$ such that the field equations remain gauge invariant and describe a gauge field with mass $\alpha$, [1]. The extra term $\mathscr{L}_{\mathrm{cs}}^{(3)}$ is known also as the Chern-Simons secondary characteristic and a general formula, valid in all odd dimensions, can be found in [2]. The Lagrangian $\mathscr{L}=\mathscr{L}_{\mathrm{ym}}+\alpha \mathscr{L}_{\mathrm{cs}}^{(3)}$ is not gauge invariant and it defines a phase factor $\Lambda(U, A)$ by $S\left(U^{-1} A U+U^{-1} d U\right)=\Lambda(U, A) \cdot S(A)$, where $S(A)=\exp \int i \mathscr{L}(A) d^{3} x$ is the action as a function of the vector potential $A$. As shown in [3], the phase factor $\Lambda$ modifies the local charge commutation relations (provided one has non-trivial boundary conditions for the gauge fields at space infinity) in such a way that the new commutators define an one dimensional central extension of the Lie algebra of infinitesimal gauge transformations (one has "Schwinger terms"), isomorphic to a Kac-Moody algebra. The central extension is determined by the boundary values at $\|\vec{x}\| \rightarrow \infty$ of the gauge fields; if we require zero boundary conditions then the extension is trivial.

In the present paper I shall extend the results to $2 n+1$ dimensions and the relation between the modified current algebra in $2 n+1$ dimensions and non-abelian chiral anomalies in $2 n$ dimensional field theories is investigated. The origin of chiral anomalies is not discussed in this paper; for a physical background a reader not familiar with anomalies is suggested to look at [5] or [6]. 
An important difference in $d=2 n+1>3$ compared to the case $d=3$ is that we have to define an infinite dimensional abelian extension of the gauge algebra in order to close the Lie algebra. Let $\mathscr{F}(\mathscr{A})$ be the space of functions $f=f(A)$ which are space integrals of polynomial functions of the components $A_{i}^{a}$ and their derivatives (with smooth coefficients). Let $X$ and $Y$ be infinitesimal time independent gauge transformations and $f, g \in \mathscr{F}(\mathscr{A})$. The extension of the gauge algebra by the abelian Lie algebra $\mathscr{F}(\mathscr{A})$ is

$$
[(X, f),(Y, g)]=\left([X, Y], \delta_{X} g-\delta_{Y} f+\alpha \theta^{(2 n)}(X, Y ; A)\right),
$$

where $\delta_{X} g$ means the Lie derivative of the function $g(A)$ with respect to the gauge transformation $X$ and $\theta^{(2 n)}$ is a certain second order cocycle (for the role of cohomology in gauge theories see [7]); in the case $n=1 \theta^{(2 n)}$ does not depend on $A$. In general, $\theta^{(2 n)}$ is antisymmetric and bilinear in the variables $X, Y$ and it is a polynomial function of $A$, satisfying the closed cocycle condition

$$
\begin{gathered}
\delta_{X} \theta^{(2 n)}(Y, Z ; A)+\delta_{Y} \theta^{(2 n)}(Z, X ; A)+\delta_{Z} \theta^{(2 n)}(X, Y ; A) \\
+\theta^{(2 n)}(X,[Y, Z] ; A)+\theta^{(2 n)}(Y,[Z, X] ; A)+\theta^{(2 n)}(Z,[X, Y] ; A) \\
=0 .
\end{gathered}
$$

Actually, $\theta^{(2 n)}(X, Y ; A)$ is a coboundary of a cocycle $\lambda_{b}^{(2 n)}(X ; A)$,

$$
\theta^{(2 n)}(X, Y ; A)=\delta_{X} \lambda_{b}^{(2 n)}(Y ; A)-\delta_{Y} \lambda_{b}^{(2 n)}(X ; A)-\lambda_{b}^{(2 n)}([X, Y] ; A),
$$

which in turn is essentially the space integral at time $x_{0}=0$ over the non-abelian anomaly in $2 n$ dimensions. At this point the reader might wonder what is wrong, since usually one assumes that the anomaly satisfies the Wess-Zumino consistency condition which says that the coboundary of $\lambda_{b}^{(2 n)}$ should be zero, $[4,5]$. In fact $\theta^{(2 n)}$ is zero if the boundary values of all fields considered are zero at $\|\vec{x}\| \rightarrow \infty$. Now $\lambda_{b}^{(2 n)}$ differs by a boundary integral from $\lambda^{(2 n)}$ which is the integral of the nonabelian anomaly $\omega^{(2 n)}$ and for which the consistency condition holds. The boundary integral has been subtracted in order to make the total abelian (central) charges equal to zero. The reason why the non-abelian anomaly appears in the commutation relation can be traced to the fact $\omega^{(2 n)}$ can be defined by $\delta_{X} \mathscr{L}_{\mathrm{cs}}^{(2 n+1)}=$ $d \omega^{(2 n)}(X ; A)$, as shown in [5].

The results are valid in a pure gauge field theory as well as in a theory of gauge fields coupled to external or dynamical sources; the difference appears only at the next step, namely in the definition of the physical states $\Psi$ (in the former case one has to require $\rho(x) \Psi=0$ and in the latter $\rho(x) \Psi=\rho_{\text {ext }}(x) \Psi$ for the charge operator $\rho(x)$ corresponding to an infinitesimal gauge transformation $X$ ).

In the last section we study the four dimensional Wess-Zumino model for a SU(3) valued field $U$. We shall see that $U$ can be coupled with an external SU(3) vector potential $A$ in a gauge invariant manner by considering the space-time as a boundary of a five dimensional manifold, extending the fields to five dimensions (the fifth dimension is in fact a homotopy parameter) and the defining the interaction through $\mathscr{L}_{\mathrm{cs}}^{(5)}\left(U^{-1} A U+U^{-1} d U\right)$; in the special case $A=0$ one gets the usual (anomalous) self-interaction for $U$. 


\section{Yang-Mills Theory with Topological Mass in $2 n+1$ Dimensions}

Let $M$ be a $2 n+1$ dimensional compact space-time and $g$ the Lie algebra of a Lie group $G$. In a gauge field theory one usually assumes $G$ to be compact and semi-simple (or at least reductive), but that does not play any role in the following. Let $\mathrm{g}^{M}$ be the Lie algebra of the group of gauge transformations: elements of $\mathrm{g}^{M}$ are smooth mappings $X: M \rightarrow \mathfrak{g}$, the commutator $[X, Y]$ is defined pointwise. We fix a matrix representation $\rho$ of $\mathfrak{g}$. In the case $G=U(n)$ the standard choice is the defining representation. Local coordinates of $M$ will be denoted by $x_{0}, x_{1}, \ldots, x_{2 n}$.

Consider the Lagrangian density

$$
\mathscr{L}=\mathscr{L}_{\mathrm{ym}}+\alpha \mathscr{L}_{\mathrm{cs}}^{(2 n+1)},
$$

where $\mathscr{L}_{\text {ym }}=(1 / 4) \operatorname{tr} \rho\left(F_{i j}\right) \rho\left(F^{i j}\right)$, in the coordinate representation (Einstein summation convention; indices are raised and lowered with respect to a fixed (pseudo-) metric on $M$ ). If $A$ is a smooth $\mathfrak{g}$-valued vector potential,

$$
F_{i j}=\partial_{i} A_{j}-\partial_{j} A_{i}+\left[A_{i}, A_{j}\right],
$$

or simply $F=d A+[A, A]$. The second term in (2.1) is a constant $\alpha$ times the Chern-Simons secondary characteristic [2], [5],

$$
\mathscr{L}_{\mathrm{cs}}^{(2 n+1)}=(n+1) \int_{0}^{1} t^{n} \operatorname{tr} A \wedge(d A+t A \wedge A)^{n} d t,
$$

where $\operatorname{tr} A B \ldots F$ is a shortened notation for $\operatorname{tr} \rho(A) \rho(B) \ldots \rho(F)$ and the $n^{\text {th }}$ power is defined with respect to the outer product $\wedge$. Assuming that the form $\langle A, B\rangle=$ $\operatorname{tr} \rho(A) \rho(B)$ is non-degenerate, the field equation derived from (2.1) is

$$
d^{*} F^{a}+\left[A,{ }^{*} F\right]^{a}+\alpha \operatorname{tr} T^{a} F^{n}=0,
$$

where $\left\{T^{1}, T^{2}, \ldots, T^{N}\right\}$ is a basis for $\mathfrak{g}$. Written in coordinates, for example in the case $n=2$,

$$
\partial^{i} F_{i j}^{a}+\left[A^{i}, F_{i j}\right]^{a}+\alpha \varepsilon_{j i k l m} \operatorname{tr} T^{a} F^{i k} F^{l m}=0,
$$

where $\varepsilon_{01234}=+1$ and $\varepsilon$ is totally antisymmetric. The trace in (2.4) gives the $n^{\text {th }}$ Chern class if $T^{a}=1$ which is also equal to the $U(1)$ anomaly in $2 n$ dimensions.

The field equation (2.4) is gauge invariant but the density $\mathscr{L}_{\mathrm{cs}}^{(2 n+1)}$ is not. If $A \mapsto U^{-1} A U+U^{-1} d U$ is a gauge transformation, then, for example in the case $n=1$,

$$
\begin{aligned}
& \mathscr{L}_{\mathrm{cs}}^{(3)}=\operatorname{tr}\left(A \wedge d A+\frac{2}{3} A \wedge A \wedge A\right), \\
& \mathscr{L}_{\mathrm{cs}}^{(3)} \stackrel{U}{\mapsto} \mathscr{L}_{\mathrm{cs}}^{(3)}+\operatorname{tr} d\left(A \wedge d U U^{-1}\right)+\frac{2}{3} \operatorname{tr}\left(d U \cdot U^{-1}\right)^{3} .
\end{aligned}
$$

Let $X$ be an infinitesimal gauge transformation, $X \in \mathfrak{g}^{M}$. If $f$ is any Frechet differentiable function of the smooth vector potential $A$, we denote by $\delta_{X} f$ the 
Frechet derivative

$$
\left(\delta_{X} f\right)(A):=\left.\frac{d}{d t} f(A+t d X+t[A, X])\right|_{t=0}
$$

From [5] (for another representation, see also [12]) we have the following general formula:

$$
\begin{aligned}
\delta_{X} \mathscr{L}_{\mathrm{cs}}^{(2 n+1)}= & d \omega^{(2 n)}(X ; A) \\
\omega^{(2 n)}(X ; A)= & -(n+1) \int_{0}^{1} \operatorname{tr} X F_{t}^{n} d t \\
& +n(n+1) \int_{0}^{1} t(t-1) \cdot \frac{1}{2} \operatorname{tr}(A \wedge[X, A]+[X, A] \wedge A) \wedge F_{t}^{n-1} d t
\end{aligned}
$$

where $F_{t}:=t d A+t^{2} A \wedge A$. The three lowest $2 n$ forms $\omega^{(2 n)}$ are, [5],

$$
\begin{aligned}
\omega^{(2)}(X ; A)= & \operatorname{tr} X d A, \\
\omega^{(4)}(X ; A):= & \operatorname{tr} X d\left(A \wedge d A+\frac{1}{2} A^{3}\right) \\
\omega^{(6)}(X ; A)= & \operatorname{tr} X d\left(A \wedge d A \wedge d A+\frac{2}{5} A^{3} \wedge d A+\frac{2}{5} d A \wedge A^{3}\right. \\
& \left.+\frac{1}{5} A^{2} \wedge d A \wedge A+\frac{1}{5} A \wedge d A \wedge A^{2}+\frac{2}{5} A^{5}\right) .
\end{aligned}
$$

The form $\omega^{(2 n)}$ is the non-abelian chiral anomaly in $2 n$ dimensions, [5]. Since

$$
\delta_{X} \int_{M} \mathscr{L}_{\mathrm{cs}}^{(2 n+1)}=\int_{M} d \omega^{(2 n)}(X ; A)=\int_{\partial M} \omega^{(2 n)}(X ; A)
$$

we have

$$
\delta_{X} \int_{M} \mathscr{L}_{\mathrm{cs}}^{(2 n+1)}=0
$$

in the case of vanishing boundary conditions for the gauge potential at space-time infinity. In this case the action

$$
S=\exp i \int_{M} \mathscr{L}
$$

is invariant with respect to "small" gauge transformations (= homotopically trivial transformations); as shown in [8] it is not invariant with respect to "large" (homotopically non-trivial) gauge transformations except when the parameter $\alpha$ is quantized according to $\alpha=$ integer $\times$ constant.

In the following we examine what happens when we have non-trivial boundary conditions. We assume

i) $M=S \times[a, b]$, where $[a, b]$ is a time interval (it may be infinite) and $S$ is the space,

ii) the choice of the gauge $A_{0}=0$,

iii) $\partial_{0} A=0$ on the space boundary $\partial S$; by ii) this is equivalent to $F_{0 k}=0$ on $\partial S$.

Let us set

$$
\lambda^{(2 n)}(X ; A)=\int_{S\left(x_{0}=\text { constant }\right)} \omega^{(2 n)}(X ; A)
$$


From (2.10) we get for a time independent $X \in \mathfrak{g}^{M}$ :

$$
\begin{aligned}
\delta_{X} \int_{M} \mathscr{L}_{\mathrm{cs}}^{(2 n+1)} & =\int_{[a, b] \times \partial S} \omega^{(2 n)}(X ; A)+\int_{S\left(x_{0}=b\right)} \omega^{(2 n)}(X ; A) \\
-\int_{S\left(x_{0}=a\right)} \omega^{(2 n)}(X ; A) & =\lambda^{(2 n)}\left(X ; A\left(x_{0}=b\right)\right)-\lambda^{(2 n)}\left(X ; A\left(x_{0}=a\right)\right) .
\end{aligned}
$$

The first integral on the right vanishes because of ii) and iii). In particular,

$$
\delta_{X} \int_{M} \mathscr{L}=0
$$

if $A\left(x_{0}=a\right)=A\left(x_{0}=b\right)$. In the general case one can recover gauge invariance of the action (2.12) by modifying the action of the Lie algebra $\mathfrak{g}^{M}$ in the space of Frechet differentiable functions of vector potentials $A$ satisfying i)-iii). The new projective representation is

$$
\tilde{\delta}_{X} f:=\delta_{X} f-i\left(\lambda^{(2 n)}\left(X ; A\left(x_{0}=b\right)\right)-\lambda^{(2 n)}\left(X ; A\left(x_{0}=a\right)\right)\right) f .
$$

In the next section we shall see how the projective factor $\lambda^{(2 n)}$ arises in the Hamiltonian formalism and how it affects the equal time commutation relations.

\section{Equal Time Commutation Relations}

Let $\mathscr{A}$ be the linear space of smooth vector potentials $A$ defined in a compact space $S$ with a smooth boundary $\partial S$, in the temporal gauge $A_{0}=0$. Physically, one might think $\partial S$ as the spatial infinity $\|\vec{x}\| \rightarrow \infty$; typically, $\partial S=S^{2 n-1}$, the $2 n-1$ dimensional sphere. Let $\mathscr{F}(\mathscr{A})$ be the linear space of functions $f(A)=\int_{S} \omega(A)$, where $\omega$ is an arbitrary differential form of degree $2 n$ which is a polynomial in the components $A_{i}^{a}$ and their derivatives, with smooth coefficients. Let us denote

$$
T \frac{\partial}{\partial A} f(A):=\left.\frac{d}{d t} f(A+t T)\right|_{t=0}
$$

where $T \in \mathscr{A}, f \in \mathscr{F}(\mathscr{A})$. Let $\mathfrak{g}^{S}$ be the Lie algebra consisting of infinitesimal time independent gauge transformations. By (2.7),

$$
\delta_{X} f=(d X+[A, X]) \frac{\partial}{\partial A} f
$$

for any $X \in \mathfrak{g}^{S}$.

The canonical equal time commutation relations

$$
\begin{aligned}
{\left[\Pi_{j}^{a}(x), A_{k}^{b}(y)\right] } & =i \delta_{j k} \delta_{a b} \delta(x-y), \\
{[\Pi(x), \Pi(y)] } & =0=[A(x), A(y)],
\end{aligned}
$$

can be represented by the smeared operators

$$
\begin{aligned}
& A(u) f)(A):=\langle A, u\rangle f(A), \\
& (\Pi(u) f)(A):=i u \frac{\partial}{\partial A} f(A),
\end{aligned}
$$


where $f \in \mathscr{F}(\mathscr{A})$ and $u \in \mathscr{A}$ is a test function,

$$
\langle A, u\rangle:=\int_{S} \operatorname{tr} A_{j} u^{j}
$$

Thus

$$
[\Pi(u), A(v)]=i\langle u, v\rangle,
$$

Let now $\Pi$ be the canonical momenta conjugate to the components of $A$ with respect to the Lagrangian

$$
L(A, \dot{A})=\int_{S} \mathscr{L}\left(x_{0}=0\right)
$$

where $\mathscr{L}=\mathscr{L}_{\mathrm{ym}}+\alpha \mathscr{L}_{\mathrm{cs}}^{(2 n+1)}$ as before and $\dot{A}:=\partial_{0} A$. The canonical definition

$$
\Pi(u):=\left.u \frac{\partial}{\partial \dot{A}} L\right|_{A_{0}=0}
$$

gives

$$
\Pi(u)=E(u)+\alpha(n+1) \int_{S}^{1} \int_{0}^{1} d t \cdot t^{n} \times \frac{1}{2} \operatorname{tr}(A u+u A)(d A+t A \wedge A)^{n-1}
$$

where

$$
E(u):=\int_{S} \operatorname{tr} F_{k 0} u^{k}
$$

The first step in quantizing the classical charge densities $\rho^{a}(x)(1 \leqq a \leqq N)$ defined by

$$
\rho^{a}=\partial^{k} F_{k 0}^{a}+\left[A^{k}, F_{k 0}\right]^{a}+\alpha \operatorname{tr} T^{a} \varepsilon_{0 i_{1} i_{2} \ldots i_{2 n}} F^{i_{1} i_{2}} \ldots F^{i_{2 n-1} i_{2 n}}
$$

(compare with (2.5)) is to write

$$
\rho(X):=\int_{S} \operatorname{tr} \rho(x) X(x), \quad X \in \mathfrak{g}^{S},
$$

in terms of the canonical variables. Note that

$$
\rho(X)=\left.X \frac{\partial}{\partial A_{0}} L\right|_{A_{0}=0}
$$

by definition. For given $X \in \mathfrak{g}^{S}$, define $Y \in \mathfrak{g}^{M}$ by $Y=x_{0} X$. Then

$$
\begin{aligned}
\left.\delta_{Y} L\right|_{X_{0}=0=A_{0}} & =\left.\left(X \frac{\partial}{\partial A_{0}}+d X \frac{\partial}{\partial \dot{A}}+[A, X] \frac{\partial}{\partial \dot{A}}\right) L\right|_{A_{0}=0} \\
& =\rho(X)+\Pi(d X+[A, X]) .
\end{aligned}
$$

On the other hand, the Yang-Mills part of $\delta_{Y} L$ is zero and the Chern-Simons term gives

$$
\begin{aligned}
\left.\delta_{Y} L\right|_{X_{0}=0=A_{0}} & \left.=\alpha \int_{S} x_{0}\right\lrcorner\left.d \omega^{(2 n)}(Y ; A)\right|_{X_{0}=0=A_{0}} \\
& =\left.\alpha \int_{S} \omega^{(2 n)}(X ; A)\right|_{A_{0}=0}=\left.\alpha \lambda^{(2 n)}(X ; A)\right|_{A_{0}=0}
\end{aligned}
$$


where

$$
\left.x_{0}\right\lrcorner \theta d x_{0} \wedge d x_{1} \wedge \ldots \wedge d x_{2 n}:=\theta d x_{1} \wedge \ldots \wedge d x_{2 n} .
$$

In (3.15) we have used the fact that

$$
d \omega^{(2 n)}(X ; A)=\operatorname{tr} d X \wedge d \Omega(A)
$$

for some $(2 n-1)$ form $\Omega$, [5], see Eqs. (2.9), and

$$
\left.x_{0}\right\lrcorner\left.\operatorname{tr} d Y \wedge d \Omega\right|_{X_{0}=0}=\operatorname{tr} X d \Omega .
$$

Combining (3.14) and (3.15) we get

$$
\rho(X)=-\Pi(d X+[A, X])+\alpha \lambda^{(2 n)}(X ; A)
$$

in the Hamiltonian formalism. Let now $X=$ constant. If $X$ is in the center of $\mathfrak{g}$ then the first term in (3.19) is zero but $\lambda^{(2 n)}(X ; A) \neq 0$. In order to make the total abelian charges equal to zero, we replace

$$
\lambda^{(2 n)} \mapsto \lambda_{b}^{(2 n)}:=\lambda^{(2 n)}-\alpha \int_{\partial S} \operatorname{tr} X \Omega(A)=-\alpha \int_{S} \operatorname{tr} d X \wedge \Omega(A),
$$

where we have used (3.17). If $X$ is constant, clearly $\lambda_{b}^{(2 n)}(X ; A)=0$. By (3.2), (3.4) and (3.19) the quantized charge operator $\hat{\rho}(X)$ is

$$
\hat{\rho}(X) f=-i \delta_{X} f+\alpha \lambda_{b}^{(2 n)}(X ; A) f,
$$

$X \in \mathfrak{g}^{S}, f \in \mathscr{F}(\mathscr{A})$, and the commutation relations are

$$
\begin{aligned}
i[\hat{\rho}(X), \hat{\rho}(Y)] & =\hat{\rho}([X, Y])+\alpha \theta^{(2 n)}(X, Y ; A), \\
\theta^{(2 n)}(X, Y ; A): & =\delta_{X} \lambda_{b}^{(2 n)}(Y ; A)-\delta_{Y} \lambda_{b}^{(2 n)}(X ; A)-\lambda_{b}^{(2 n)}([X, Y] ; A),
\end{aligned}
$$

$X, Y \in \mathfrak{g}^{S}$. For example,

$$
\begin{aligned}
& \theta^{(2)}(X, Y ; A)=2 \int_{S} \operatorname{tr} d X \wedge d Y=2 \int_{\partial S} \operatorname{tr} X d Y, \\
& \theta^{(4)}(X, Y ; A)=2 \int_{S} \operatorname{tr} d X \wedge d Y \wedge d A=2 \int_{\partial S} \operatorname{tr} X d Y \wedge d A .
\end{aligned}
$$

In general, for $n>1 \theta^{(2 n)}$ depends on $A$. In the case $n=1$ the cocycle $\theta^{(2)}$ defines a one dimensional central extension of the Lie algebra $\mathfrak{g}^{S}$ and $(X, t) \rightarrow i \hat{\rho}(X)+t$ is a true representation of the extension $\mathrm{g}^{S}+\mathbb{R}$, [3]. Let $n>1$. Consider the space $\mathfrak{g}^{S}+\mathscr{F}(\mathscr{A})$ equipped with the commutator

$$
[(X, f),(Y, g)]:=\left([X, Y], \theta^{(2 n)}(X, Y ; .)+\delta_{X} g-\delta_{Y} f\right) .
$$

Since by (3.22) $\theta^{(2 n)}$ is a coboundary, it satisfies

$$
\begin{aligned}
& \theta^{(2 n)}(X,[Y, Z] ; A)+\theta^{(2 n)}(Z,[X, Y] ; A)+\theta^{(2 n)}(Y,[Z, X] ; A) \\
& \quad+\delta_{X} \theta^{(2 n)}(Y, Z ; A)+\delta_{Y} \theta^{(2 n)}(Z, X ; A)+\delta_{Z} \theta^{(2 n)}(X, Y ; A)=0 .
\end{aligned}
$$

which implies the Jacobi identity for the commutator (3.24), and thus (3.24) really 
defines a Lie algebra. The mapping

$$
(X, f) \mapsto i(\hat{\rho}(X)+f)
$$

defines a representation of the Lie algebra $\mathfrak{g}^{S}+\mathscr{F}(\mathscr{A})$ in the space $\mathscr{F}(\mathscr{A})$.

\section{The Chern-Simons Term in the Wess-Zumino Model}

In this section we assume that the space-time $M$ is a four dimensional compact manifold without boundary (e.g. $S^{4}$ ). The Wess-Zumino Lagrangian for a smooth field $U: M \rightarrow \mathrm{SU}(3)$ is

$$
L=-\frac{F_{\pi}^{2}}{16} \int \operatorname{tr} \partial_{\mu} U \partial^{\mu} U^{-1} d^{4} x+\mathscr{J}
$$

where $F_{\pi}$ is a constant (in QCD $F_{\pi} \simeq 190 \mathrm{MeV}, U$ describes, in the low energy limit, idealized massless kaons and pions, $[4,9])$ and $\mathscr{J}$ is chosen in such a way that the field equation derived from (4.1) is

$$
\partial^{\mu}\left(\frac{F_{\pi}^{2}}{8} U^{-1} \partial_{\mu} U\right)+\lambda \varepsilon^{\mu \nu \alpha \beta} U^{-1} \partial_{\mu} U U^{-1} \partial_{\nu} U U^{-1} \partial_{\alpha} U U^{-1} \partial_{\beta} U=0
$$

where $\lambda$ is a constant. We shall assume that the space-time $M$ is such that any map $U: M \rightarrow \mathrm{SU}(3)$ is homotopic to a constant; for example $M=S^{4}$, since $\pi_{4} \mathrm{SU}(3)=0$. For a given $U$, let $t \rightarrow U_{t}$ be the homotopy: $U_{t}(x)$ is smooth in $[0,1] \times M$ and $U_{0} \equiv 1, U_{1}=U$. Now $U_{t}^{-1} d U_{t}$ can be considered as a $g$-valued 1 -form on $[0,1] \times M$ and we can set

$$
\mathscr{J}=-\lambda \int_{[0,1] \times M} \operatorname{tr}\left(U_{t}^{-1} d U_{t}\right)^{5}
$$

This is a slight reformulation of the Lagrangian given in [9]. As shown in [9], the integral over $[0,1] \times M$ can be transformed into a boundary integral over $M$ and the value $\mathscr{J}(U)$ does not depend on the chosen homotopy. In particular, if $U(x)$ can be written in the form $U(x)=\exp Z(x)$ for some smooth $g$-valued mapping $Z$, we have the following explicit formula: Choosing

$$
U_{t}=\exp t Z
$$

and using the Magnus formula, [10],

$$
e^{-Z} d e^{Z}=\sum_{n=0}^{\infty} \frac{1}{(n+1) !}(-a d Z)^{n} d Z
$$

we get

$$
\mathscr{J}=\lambda \int_{M} \operatorname{tr} Z \sum_{\left(n_{i}\right)} \prod_{i=1}^{4} \frac{1}{\left(n_{i}+1\right) !} \cdot\left(2+\sum_{j=1}^{4} n_{j}\right)^{-1} \times(-a d Z)^{n_{i}} d Z .
$$

The integration over $t$ has been carried out (compare this result with the formula given in [11] for the two dimensional case). 
According to the formula (2.3),

$$
\mathscr{L}_{\mathrm{cs}}^{(5)}=\operatorname{tr}\left[A \wedge(d A)^{2}+\frac{3}{2} A^{3} \wedge d A+\frac{3}{5} A^{5}\right] .
$$

In particular, if $A=U_{t}^{-1} d U_{t}$,

$$
\mathscr{L}_{\mathrm{cs}}^{(5)}\left(U_{t}^{-1} d U_{t}\right)=\frac{1}{10} \operatorname{tr}\left(U_{t}^{-1} d U_{t}\right)^{5}
$$

and therefore

$$
\mathscr{J}(U)=-10 \lambda \int_{[0,1] \times M} \mathscr{L}_{\mathrm{cs}}^{(5)}\left(U_{t}^{-1} d U_{t}\right) .
$$

One can now define a gauge invariant interaction of $U$ with an external $\mathrm{SU}(3)$ vector potential $A$ by setting

$$
\mathscr{I}(U, A):=-10 \lambda \int \mathscr{L}_{\mathrm{cs}}^{(5)}\left(U_{t}^{-1} A U_{t}+U_{t}^{-1} d U_{t}\right) .
$$

For any gauge transformation $V: M \rightarrow \mathrm{SU}(3), \mathscr{J}(U, A)$ is invariant with respect to

$$
U_{t} \mapsto V_{t}^{-1} U_{t}, \quad A \mapsto V_{t}^{-1} A V_{t}+V_{t}^{-1} d V_{t},
$$

where $t \rightarrow V_{t}$ is a homotopy, $V_{0} \equiv 1$ and $V_{1}=V$. By a straightforward (but tedious) computation

$$
\begin{aligned}
\mathscr{L}_{\mathrm{cs}}^{(5)}\left(U_{t}^{-1} d U_{t}+U_{t}^{-1} A U_{t}\right)= & \mathscr{L}_{\mathrm{cs}}^{(5)}(A)+d \operatorname{tr}\left[-d U_{t} U_{t}^{-1} \wedge\left(A \wedge d A+\frac{1}{2} A^{3}\right)\right. \\
& -\frac{1}{2}\left(d U_{t} U_{t}^{-1}\right)^{2} \wedge A^{2}+\frac{1}{4}\left(d U_{t} U_{t}^{-1} \wedge A\right)^{2} \\
& \left.+\frac{1}{2}\left(d U_{t} U_{t}^{-1}\right)^{3} \wedge A\right]+\frac{1}{10} \operatorname{tr}\left(d U_{t} U_{t}^{-1}\right)^{5}
\end{aligned}
$$

The first term $\mathscr{L}_{\mathrm{cs}}^{(5)}(A) \equiv 0$, since the restriction of a 5 -form to the four dimensional manifold vanishes. Therefore,

$$
\begin{aligned}
\mathscr{J}(U, A)= & -10 \lambda \int_{M} \operatorname{tr}\left[-d U \cdot U^{-1} \wedge\left(A \wedge d A+\frac{1}{2} A^{3}\right)+\cdots\right] \\
& -\lambda \cdot \int_{[0,1] \times M} \operatorname{tr}\left(d U_{t} U_{t}^{-1}\right)^{5} .
\end{aligned}
$$

This is not gauge invariant! The reason is, that in (4.11) the correct form of the gauge transformation is $A \mapsto V_{t}^{-1} A V_{t}+V_{t}^{-1} d V_{t}$ and not $A \rightarrow V^{-1} A V+V^{-1} d V$; the first term $\mathscr{L}_{\mathrm{cs}}^{(5)}(A)$ in (4.11) is not gauge invariant under the former transformation. However, the non-invariance does not affect the field equation for $U$, since $\mathscr{L}_{\mathrm{cs}}^{(5)}\left(V_{t}^{-1} A V_{t}+V_{t}^{-1} d V_{t}\right)$ does not depend on $U$. Thus one can safely use the expression (4.12) for the derivation of the (gauge invariant) field equation for $U$. In the case $A=0(4.12)$ reduces to the anomalous part of the Wess-Zumino Lagrangian.

If we put $U=\exp Z$ and compute $\mathscr{J}(U, A)$ to the first order in $Z$, we get

$$
\mathscr{J}(U, A)=-10 \lambda \int_{M} \operatorname{tr}-d Z \wedge\left(A \wedge d A+\frac{1}{2} A^{3}\right)+\cdots,
$$

which is the non-abelian anomaly in four dimensions, Eq. (2.9), modulo the factor $-10 \lambda$ and an integration by parts. The usefulness of the formula (4.12) lies in the fact that it allows a straight-forward computation of the anomalous terms to all orders in $Z$ in the (left) gauged SU(3) chiral model. 
Acknowledgements. I wish to thank Christofer Cronström and Roman Jackiw for fruitful discussions. After completing this work I was told by R. Jackiw about ref. [13] where similar conclusions for the Schwinger terms were obtained in a different way.

\section{References}

1. Jackiw, R., Templeton, S.: How super-renormalizable interaction cure their infrared divergences. Phys. Rev. D23, 2291 (1981); Schonfeld, J.: A mass term for three-dimensional gauge fields. Nucl. Phys. B185, 157 (1981)

2. Chern, S., Simons, J.: Characteristic forms and geometric invariants. Ann. Math. 99, 48 (1974)

3. Mickelsson, J.: On a relation between massive Yang-Mills theories and dual string models. Lett. Math. Phys. 7, 45 (1983)

4. Wess, J., Zumino, B.: Consequences of anomalous Ward identities. Phys. Lett. 37B, 95 (1971)

5. Zumino, B., Wu Yong-Shi, Zee, A.: Chiral anomalies, higher dimensions and differential geometry. Preprint 40048-18 P3, Berkeley, California (1983)

6. Jackiw, R.: Topological investigations of quantized gauge theories. Lectures in Les Houches, JulyAugust 1983; MIT-preprint CTP No. 1108 (1983)

7. Bonora, L., Cotta-Ramusino, P.: Some remarks on BRS transformations, anomalies and the cohomology of the Lie algebra of the group of gauge transformations. Commun. Math. Phys. 87, 589 (1983)

8. Deser, S., Jackiw, R., Templeton, S.: Three-dimensional massive gauge theories. Phys. Rev. Lett. 48, 975 (1982); Topologically massive gauge theories. Ann. Phys. (NY) 140, 372 (1982)

9. Witten, E.: Global aspects of current algebra. Preprint, Princeton (1983)

10. Magnus, W.: On the exponential solution of differential equations for a linear operator. Commun. Pure Appl. Math. 7, 649 (1954)

11. Cronström, C., Mickelsson, J.: On topological boundary characteristics in nonabelian gauge theory. J. Math. Phys. 24, 2528 (1983)

12. Frampton, P. H., Kephart, T. W.: Analysis of anomalies in higher space-time dimensions. Phys. Rev. D28, 1010 (1983); Frampton, P. H., Kephart, T. W.: Explicit evaluation of anomalies in higher dimensions. Phys. Rev. Lett. 50, 1343 (1983); Frampton, P. H., Kephart, T. W.: Consistency conditions for Kaluza-Klein anomalies. Phys. Rev. Lett. 50, 1347 (1983)

13. Faddeev, L. D.: Operator anomaly for the Gauss law. Phys. Lett. 145B, 81 (1984)

Communicated by S.-T. Yau

Received December 15, 1983; in revised form June 5, 1984 